\title{
Téoros
}

Revue de recherche en tourisme

\section{À la recherche du patrimoine de Hong Kong : grandeur et complexité}

\section{Martin Drouin}

Volume 27, numéro 2, été 2008

URI : https://id.erudit.org/iderudit/1070799ar

DOI : https://doi.org/10.7202/1070799ar

Aller au sommaire du numéro

\section{Éditeur(s)}

Université du Québec à Montréal

ISSN

0712-8657 (imprimé)

1923-2705 (numérique)

Découvrir la revue

\section{Citer cette note}

Drouin, M. (2008). À la recherche du patrimoine de Hong Kong : grandeur et complexité. Téoros, 27(2), 63-66. https://doi.org/10.7202/1070799ar 


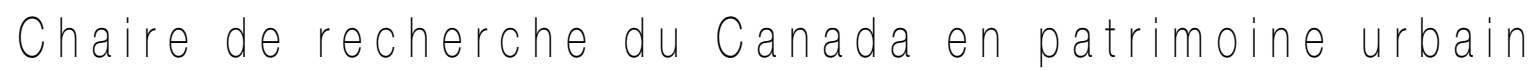 \\ À la recherche du patrimoine de Hong Kong : grandeur et complexité}

\section{Martin Drouin}

Hong Kong s'impose comme une destination incontestable dans l'orbite des villes touristiques de calibre international. Au premier abord cependant, peu de touristes choisiraient d'y passer une partie de leur séjour afin de pouvoir goûter au patrimoine urbain hongkongais. Face à la richesse architecturale de Macao, l'autre port au passé européen du delta de la rivière des Perles, la ville ferait en effet pâle figure. Dans ma chronique précédente, j'évoquais les transformations récentes de l'ancienne colonie portugaise, dont l'inscription en 2005 du centre historique sur la liste du patrimoine mondial de l'Organisation des Nations Unies pour l'éducation, la science et la culture (UNESCO), qui en faisait un lieu prisé pour la qualité de son architecture ancienne. Je soulignais par la même occasion l'émergence d'un inévitable paradoxe qui oppose la mise en tourisme de ce patrimoine, valorisé par la valeur d'authenticité, et la construction d'une série de casinos, associée davantage à la poursuite de l'illusion et du trompe-l'œil. Le développement touristique de Hong Kong ne s'inscrit assurément pas dans une même dynamique. L'ancien "rocher stérile » décrit par Lord Palmerston après la signature du traité de Nankin en 1842 a bien d'autres attraits à offrir. Le petit port de pêche, cédé au Royaume-Uni pendant une période de 155 ans, s'est transformé en l'un des centres financiers les plus influents d'Asie. II fait désormais partie de ces villes monde au cœur de la reconfiguration des économies actuelles. Le transfert de la colonie à la Chine - le « handover » comme les événements de 1997 sont communément appelés - n'a rien changé au dynamisme local. Dans le brouhaha d'une telle effervescence, l'identité de la ville s'est articulée autour de ses nombreux gratte-ciel et de ses néons. Cette image d'Épinal se retrouve encore sur les cartes postales vendues aux touristes de passage. La "symphonie des lumières » est d'ailleurs le nom d'un spectacle donné tous les soirs, auquel participent 44 édifices de la ville dans un feu d'artifice lumineux ${ }^{1}$. Dans ce contexte, une offre touristique pourrait-elle franchement miser sur le patrimoine hongkongais ?

Le caractère international de la ville et le dynamisme qui l'accompagne ont été choisis par le Hong Kong Tourist Board (HKTB), organisme gouvernemental créé en 2001, pour construire la personnalité touristique de la ville ${ }^{2}$. "Hong Kong, Live it, Love it! » : telle est la formule aujourd'hui scandée au visiteur sur les brochures officielles (HKTB, 2001-2006). La stratégie de marketing

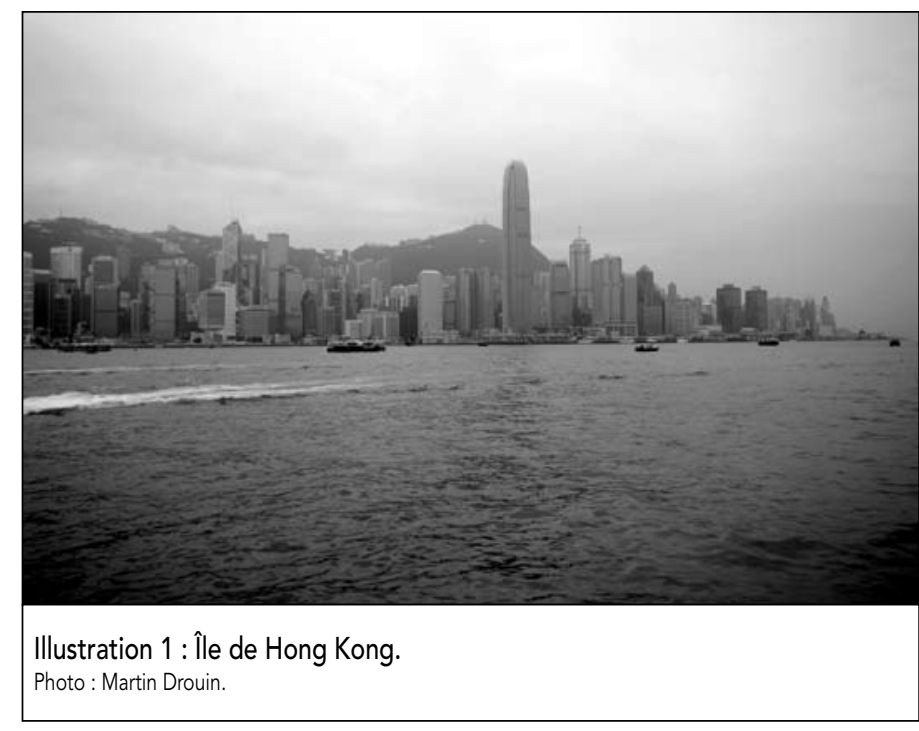

se décline autour de quatre " produits piliers » - qui ont émergé après d' « intensives recherches »-, afin de positionner la ville sur le marché mondial du tourisme. L'observateur comprendra aisément, tout d'abord, que des stratèges aient pu miser sur la "position géographique » de la ville. En effet, la conjugaison des éléments naturels et topographiques, à partir desquels la ville s'est construite, fait partie intégrante de l'expérience du visiteur. Un simple trajet entre les différentes parties de la ville émerveille même le plus blasé des globe-trotters. II acquiescera, ensuite, à l'idée d'avoir parié sur le "shopping ». Hong Kong rassemble à la fois des marchés traditionnels et des galeries entières de boutiques de luxe. Dans les premiers, le consommateur peut marchander et enfin sourire à l'idée d'acheter des produits locaux, fabriqués en Chine. Dans les secondes, il peut s'extasier devant les grandes marques de la planète mode. Entre le vrai et le faux, Hong Kong approvisionne les clients les plus sourcilleux. L'observateur approuvera également le choix d'avoir souligné les ressources reliées aux " plaisirs gourmands » de la ville. Comme pour le « produit pilier » précédent, un florilège de possibilités s'offre à lui, de la plus petite échoppe de nouilles à la haute gastronomie. Hong Kong se targue d'ailleurs d'être l'une des capitales culinaires de l'Asie. Enfin, il cautionnera l'idée de mettre en valeur la " culture ". D'événements en festivals, des institutions muséales et culturelles à Disneyland et au village de Ngong Ping sur l'île de Lantau, la ville 


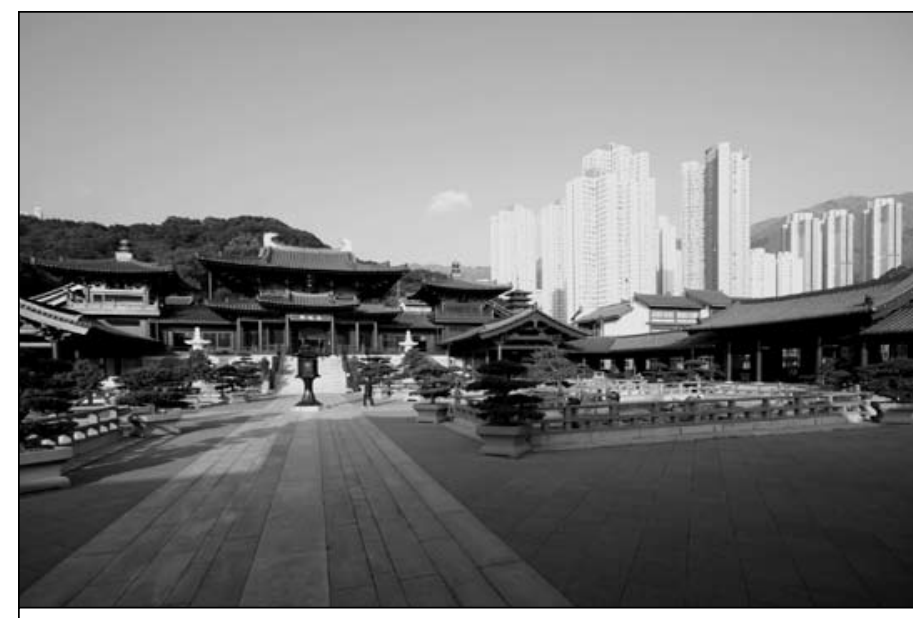

Illustration 2 : Nan Lian Garden, Hong Kong.

Photo : Martin Drouin.

se présente tel un « kaléidoscope " réfléchissant des combinaisons d'expériences. L'observateur s'interrogera cependant, surtout s'il a fait une escale à Macao, sur ce qui se cache sous l'étiquette de « patrimoine » qui a été associée à la notion de culture.

Le paysage urbain de Hong Kong s'est énormément transformé au cours de la seconde moitié du $X X^{\ominus}$ siècle. Cette ville, dont l'économie a véritablement décollé au lendemain de la Seconde Guerre mondiale et de la naissance de la République populaire de Chine en 1949, est devenue depuis l'un des quatre "dragons asiatiques $^{3}$ ". L'impact de cette croissance et l'urbanisation qui s'en est suivie sont plus que manifestes sur le territoire de 1104 kilomètres carrés, dont la population a atteint aujourd'hui près de 7 millions d'habitants, faisant de Hong Kong l'une des villes les plus densément peuplées au monde. Dans ce contexte, la conservation du patrimoine n'a pas été une priorité. En repoussant à 1800 la date charnière à laquelle des bâtiments peuvent être jugés exceptionnels, les législateurs donnaient toute la latitude nécessaire pour juger les protections au cas par cas ${ }^{4}$. De la démolition de l'ancien bureau de poste au démantèlement du terminal du Star Ferry et du quai Queen's, en passant par la disparition de la ville fortifiée de Kowloon, les exemples abondent (Abbas, 1997 ; Harter, 2000 ; Chui Hau Man et Tsoi Tan Mei, 2003). Les remblaiements successifs des deux côtés du port de Victoria, en plus de causer des torts irréparables aux écosystèmes, posent de sérieux problèmes à l'animation portuaire. II n'est peut-être pas si loin le moment où ce port mythique ne sera plus qu'un souvenir, tout comme les avions surplombant la ville quelques minutes avant l'atterrissage appartiennent désormais à une sorte de folklore quasi impensable. II ne s'agit pas ici de sombrer dans la nostalgie, mais simplement de noter le peu de gestes posés pour sauvegarder ou soustraire la ville aux diktats de la croissance économique. Les anciennes autorités coloniales n'ont pas mieux fait que leurs successeurs chinois. Les premières trouvèrent plus de gloire à laisser en héritage un nouvel aéroport ultramoderne sur l'île de Lantau (Lee et du Cros, 2007), tandis que les seconds, dans un contexte de décolonisation, ont préféré ne pas trop insister sur les traces de l'ancienne ville britannique (Henderson, 2001). Les deux puissances souhaitaient davantage s'associer au dynamisme économique hongkongais pour en revendiquer la paternité 5 .

Aux détracteurs de la politique d'aménagement urbain, des esprits malicieux seraient peut-être tentés de pointer la silhouette de la ville pour trouver un patrimoine à montrer aux touristes. Que le visiteur regarde d'un côté ou de l'autre du port de Victoria, tant en direction de l'île de Hong Kong que vers Kowloon, il est immanquablement impressionné par la succession et la surimposition d'immeubles en hauteur. L'incontournable ascension que font tous les touristes au sommet du mont Victoria - au Peak - permet de mieux en mesurer, de jour comme de nuit, l'incroyable densité. Les grandes villes occidentales où poussent les forêts de gratte-ciel pourraient bien pâlir d'envie. Les autres mégapoles d'Asie, que ce soit Singapour, Kuala Lumpur ou Tokyo, se posent en simples compétiteurs, rien de plus. D'ailleurs, selon certaines compilations, Hong Kong arriverait première au monde pour le nombre de gratte-ciel (7687), loin devant sa rivale étatsunienne, New York, avec ses 5633 édifices (Emporis, 2008). La ville serait aussi la deuxième plus haute ville du monde derrière Chicago (Ultrapolis Project, 2007). De plus, des architectes de renommée internationale y ont signé plusieurs bâtiments prestigieux : la tour HSBC de Normand Foster, la banque de Chine de leoh Ming Pei, le Hong Kong Convention and Exibition Centre de SOM (Skidmore, Owings, Merrill) et le Lippo Centre de Paul Rudolph, pour n'en nommer que quelques-uns. À l'opposé des œuvres signées, une architecture sans nom s'est affirmée en s'appropriant - par addition, juxtaposition et une grande dose de bricolage - les édifices d'habitation en hauteur pour en faire une véritable expression du vernaculaire. Ces habitations organiques trouvent aussi leur place dans les guides d'architecture de Hong Kong : "S'il fallait résumer par un seul type de bâtiment la quintessence architecturale de cette ville, ce serait sans doute ce mode d'expression libre, spontané et anarchique par lequel les habitants s'approprient le bâti. » (Cheung et Yeoh, 1998) Façades lumineuses ou de verre poli, façades d'une complexité confuse et luxuriante, il est clair que les gratte-ciel et les autres édifices en hauteur seront un patrimoine de demain.

Aux yeux des promoteurs touristiques, le patrimoine se trouve pourtant ailleurs. II serait même complètement hors du centreville. II se cache ou s'épanouit - c'est selon les points de vue - dans les Nouveaux Territoires. Comme leur nom l'indique, il s'agit originellement de nouvelles concessions octroyées par la Chine à la fin du XIXe siècle et situées sur le continent dans l'arrière-pays du territoire de Kowloon. À partir du milieu des années 1980, des monuments localisés dans les Nouveaux Territoires ont été restaurés et mis en valeur par l'Antiquities and Monuments Office, organe gouvernemental responsable de la protection du patrimoine. Menée dans une optique d'éducation populaire mais aussi de développement touristique, l'entreprise souhaitait valoriser l'unicité du patrimoine culturel de Hong Kong (Cheung, 2003). Une décennie plus tard, des circuits patrimoniaux étaient dessinés afin de relier entre eux les différents sites pour en faire une offre attrayante. Depuis, les visiteurs foulent la Ping Shang Heritage Trail 
(Cheung, 1999). Au nombre des attractions se trouvent la plus ancienne pagode de Hong Kong, I'un des plus grands halls du clan des Tang, un village fortifié, une ancienne école pour éduquer les futurs fonctionnaires au service de la Chine impériale et un temple dédié à Hung Shing (Antiquities and Monuments Office, 2004-2008). Un deuxième circuit a été créé quelques années plus tard, toujours dans les Nouveaux Territoires. Encore une fois, le choix des lieux à visiter célèbre l'héritage précolonial. Ces circuits tentent ainsi de proposer un contraste bien réel entre l'Occident et l'Orient, entre les mondes moderne et traditionnel, entre un paysage urbanisé et une nature palpable, entre le tangible et l'intangible. Dans les mêmes secteurs et en parallèle de ces initiatives, un patrimoine vert émerge même. Aux plages de Repulse Bay et de Stanley, classiques de la villégiature au temps des Britanniques, répond désormais le Hong Kong Wetlands Park. À coup de millions de dollars, le projet a été mené par l'Agriculture, Fisheries and Conservation Department (ministère de l'agriculture et de la pêche) et le Hong Kong Tourist Board pour en faire une réserve naturelle en milieu humide doublée d'une attraction touristique interactive et multimédia. À l'évidence, le patrimoine ne se trouve pas là où l'on aurait pensé le rencontrer.

Un double paradoxe s'impose à l'observateur en regard de la mise en tourisme du patrimoine hongkongais. II est à la fois géographique et chronologique, mais renvoie à une seule et même problématique : celle de la ville coloniale. Contrairement à Macao où le legs portugais constitue l'essentiel de la ressource patrimoniale valorisée, un regard extrêmement large est posé à Hong Kong. Celui-ci scrute à l'extérieur du centre-ville, dans les marges, vers les Nouveaux Territoires, dans un lieu qui est « older and more traditional in character than anywhere in Kowloon or Hong Kong Island " (Ingham, 201). Ce regard considère semblablement l'aventure humaine hongkongaise s'échelonnant sur plusieurs millénaires. L'exposition permanente du Hong Kong Museum of History le démontre éloquemment. Divisée en huit salles réparties sur deux niveaux, dans un espace totalisant 7000 mètre carrés, l'histoire de la ville se raconte depuis la préhistoire (Ting et Pang, 2006). Rien de moins ! Ossements, pointes de silex et poteries exposés en sont la preuve tangible. Dans les espaces les plus impressionnants dédiés à l'environnement naturel et à la culture, la succession des événements s'efface au profit d'un temps long, presque immobile. Une approche anthropologique de quatre groupes ethniques hongkongais - les Boat Dwellers, les Punti, les Hoklo et les Hakka - présente des modes de vie traditionnels presque encore chauds d'actualité. La présence, grandeur nature, d'une jonque, d'une maison de paysan ou d'un théâtre de marionnettes - parmi les nombreux dispositifs muséographiques imaginés - rend le discours encore plus réel. L'aventure coloniale se trouve au deuxième étage avec la guerre de l'opium, l'occupation japonaise, la métropole moderne et le retour de Hong Kong à la Chine. La coupure se fait emblématique avec les événements survenus depuis la seconde moitié du XIXe siècle. La narration de l'histoire est des plus limpides et se résume ainsi : l'aventure hongkongaise s'échelonne sur 6000 ans, dont seulement 155 ans de présence britannique.

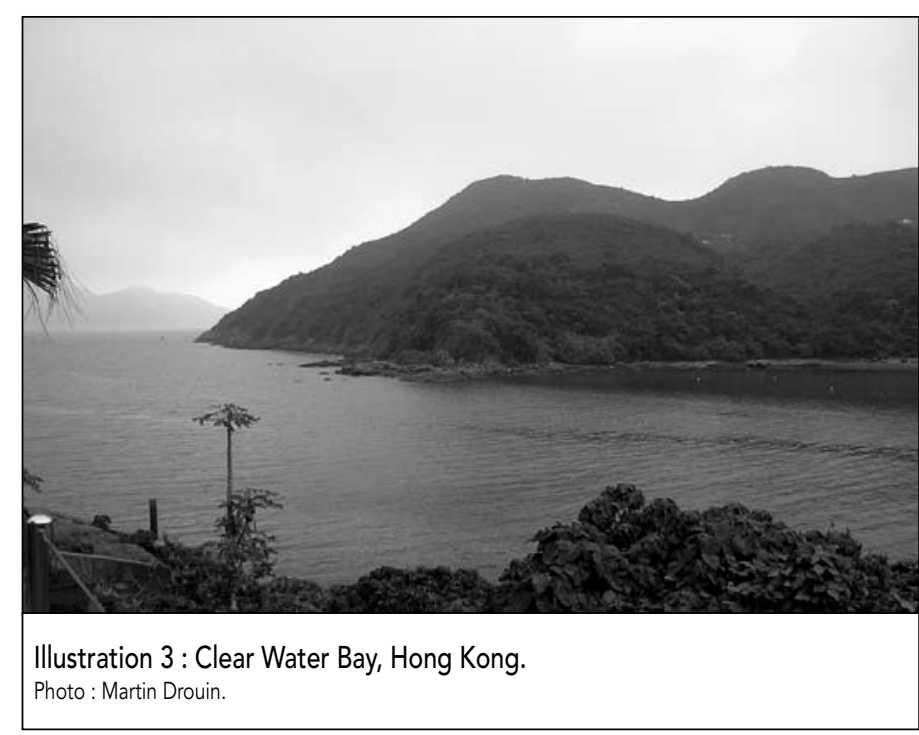

Dans le contexte de la constitution d'une offre touristique, ce constat permet de bonifier considérablement l'expérience patrimoniale et de surprendre le visiteur. Hong Kong se laisse ainsi découvrir par des facettes insoupçonnées et ne se résume pas à la partie le plus congrue de son histoire. II ne faudrait pas pour autant en oublier un passé plus récent. L'aventure coloniale singularise ce qui pourrait simplement se rattacher à une histoire plus générale du sud de la Chine. Des circuits patrimoniaux ayant pour thème la période britannique ont d'ailleurs été mis en place ces dernières années sur l'île de Hong Kong. Malheureusement, trop souvent, beaucoup d'attraits se résument à une plaque commémorative, l'édifice ayant été démoli ou le lieu considérablement transformé. Les protestations récentes et la participation des Hongkongais au processus de construction du patrimoine apparaissent de bon augure pour les prochaines années. Les manifestations récentes à propos de la démolition du terminal du Star Ferry l'ont démontré, tout comme les efforts pour préserver le quartier s'articulant autour de la rue Lee Tung, mieux connue sous le nom de Wedding Card Street (England, 2006). La présence de la Conservancy Association, de la Society for Protection of the Harbour et de SOS Save Our Shorelines démontre que le patrimoine de demain ne sera pas fait d'une seule vision imposée par les autorités. Les circuits de visite organisés par le premier groupe mentionné dans les rues du centre-ville en proposent d'ores et déjà une autre vision (So, 2007). La démarche s'inscrit plus généralement dans un processus de rupture et de recherche d'identité. S'il fut plus facile d'identifier le patrimoine de Macao, l'exercice s'avère beaucoup plus tortueux à Hong Kong. Ni vraiment Britanniques, ni tout à fait Chinois, les Hongkongais, tout comme le patrimoine qui témoigne de leur présence, se situent dans une expérience réelle de métissage dont les fils de l'écheveau apparaissent parfois distinctement, mais s'entremêlent la plupart du temps. La reconnaissance de cette complexité ne se fera pas facilement, mais sera, à n'en pas douter, une escale touristique riche et fascinante, loin d'une instrumentalisation du patrimoine qui pourrait être l'avenir d'autres villes de la région.

Martin Drouin est professeur associé au Département d'études urbaines et touristiques de l'UQÀM. 


\section{Notes}

1 Le spectacle a remporté le titre de "Largest Permanent Light and Sound Show » octroyé par les records Guinness (Tourism Commission, 2005).

2 L'organisme a ainsi remplacé la Hong Kong Tourist Association, ancienne association touristique locale fondée en 1957 (Li et Lo, 2004).

3 Les économistes font référence aux nouveaux pays industrialisés (Corée du Sud, Taïwan, Hong Kong et Singapour) auxquels font maintenant concurrence les "tigres asiatiques ", c'est-à-dire les nouveaux pays exportateurs dont font partie le Brunei, l'Indonésie, la Malaisie, les Philippines et la Thaillande (Nguyen, 2006).

4 La première législation pour la protection des monuments historiques fut adoptée en 1971 (Du Cros et al., 2007).

5 En 1999, deux ans après le passage de Hong Kong à la Chine, les autorités chinoises dressaient un obélisque commémoratif sur lequel on peut lire: "The people of Hong Kong, with their diligence, vigour and entrepreneurial skills, and supported by the motherland, have achieved great success in their endeavours and have turned Hong Kong into an international centre of finance, trade and transportation. " (Teather et Chow, 2003) Peu de traces du Royaume-Uni dans ce portrait.

\section{Bibliographie}

Abbas, A. (1997), Hong Kong: Culture and Politics of Disappearance, Minneapolis, University of Minneapolis Press.

Antiquities and Monuments Office, Leisure and Cultural Services Department, Government of Hong Kong (2004-2008), Site Internet officiel, [www. amo.gov.hk/en/about.php], consulté le 8 mai 2008.

Cheung, S.C.H. (2003), "Remembering Through Space: the Politics of Heritage in Hong Kong ", International Journal of Heritage Studies, vol. $9, \mathrm{n}^{\circ} 1$, p. $7-26$

Cheung, S.C.H. (1999), "The Meaning of a Heritage Trail in Hong Kong », Annals of Tourism Research, vol. 26, n 3, p. 570-588.

Cheung, J. et A. Yeoh (1998), Hong Kong. Guide de l'architecture contemporaine, Londres, Ellipses.

Chui Hau Man, M. et A. Tsoi Tan Mei (2003), « Heritage Preservation: Hong Kong \& Overseas Experiences », The Conservancy Association Officiel Web Site, [http://www.conservancy.org.hk/heritage/Heritage_Report_ eng.pdf], consulté le 8 mai 2008.
Du Cros, H., F.L. Yok-shiu, A. Sauvigrain-McClelland, E. Chow et D. Lung (2007), " The Pearl River Delta. One Region, Three systems », dans H. du Cros et Y.F. Lee, (dir)., Cultural Heritage Management in China. Preserving the Cities of the Pearl River Delta, New York, Routledge, p. 23-47.

Emporis (2008), Most Active Cities in Terms of High-rise Construction, [http:// www.emporis.com/en/bu/sk/st/ma/ci/], consulté le 10 mai 2008.

England, V. (2006), "Hong Kong Wakes Up to Heritage ", BBC News, 4 décembre, [http://news.bbc.co.uk/1/hi/world/asia-pacific/6206116. stm], consulté le 8 mai 2008.

Harter, S. (2000), " Hong Kong's Dirty Little Secret. Clearing the Walled City of Kowloon », Journal of Urban History, vol. 27, no 1, p. 92-112.

Henderson, J. (2001), "Heritage, Identity and Tourism in Hong Kong ", International Journal of Heritage Studies, vol. 7, n’ 3, p. 219-235.

Hong Kong Tourist Board (HKTB) (2001-2006), Discover Hong Kong, [www. discoverhongkong.com], consulté le 10 mai 2008.

Ingham, M.I. (2007), Hong Kong. A Cultural History, Oxford, Oxford University Press.

Lee, Y.F. et H. du Cros (2007), "The Human Factor and Cultural Affinity ", dans H. du Cros et Y.F. Lee, (dir.), Cultural Heritage Management in China. Preserving the Cities of the Pearl River Delta, New York, Routledge, p. 117-137.

Li, Y. et L.B.R. Lo (2004), «Opportunities and Constraints of Heritage Tourism in Hong Kong's Changing Cultural Landscape ", Tourism and Hospitality Research, vol. 5, n 4, p. 322-345.

Nguyen, É. (2006), L'Asie géopolitique : de la colonisation à la conquête du monde, Paris, Levallois-Perret, Groupe Studyrama.

So, P. (2007), "Tours Explores Fast-disappearing Culture », South China Morning Post, 23 décembre, p. 3.

Teather, E.K. et C.S. Chow (2003), "Identity and Place: the Testament of Designated Heritage in Hong Kong ", International Journal of Heritage Studies, vol. 9, n² 2, p. 93-115.

Ting, J.S.P. et M.W.L. Pang (dir.) (2006 [2éd.]), Hong Kong Museum of History. Brief Guide, Hong Kong, Leisure and Cultural Services Department.

Tourism Commission (2005), A Symphony of Lights, [www.tourism.gov.hk/ symphony/], consulté le 10 mai 2008.

Ultrapolis Project (2007), Tallest Cities in the World, [http://www.ultrapolisproject.com/ultrapolis_world_tallest_skylines_cities.htm], consulté le 10 mai 2008.

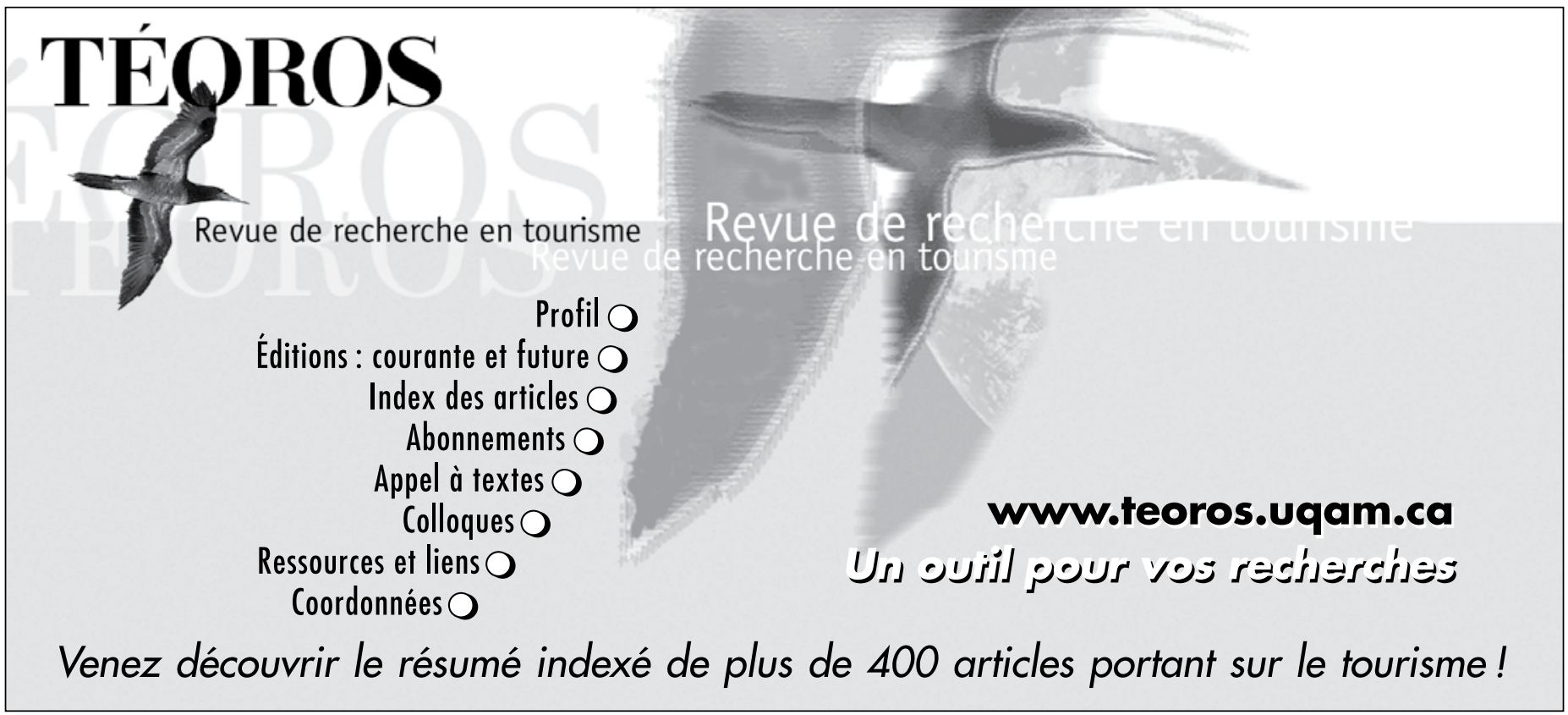

